\title{
'ROSE to DELAROSE' - Developing and transitioning a web- based pan European self-help programme for the management of work related stress.
}

\author{
J.S.G. Wells*, C. Ryan** \& M. Bergin*** \\ * Head of School of Health Sciences, Waterford Institute of Technology, Waterford, Ireland, \\ jswells@wit.ie \\ ** Research Assistant, School of Health Sciences, Waterford Institute of Technology, Waterford, \\ Ireland, cryan@wit.ie \\ *** Lecturer, Department of Nursing \& Healthcare, School of Health Sciences, Waterford \\ Institute of Technology, Waterford, Ireland, mbergin@wit.ie
}

\begin{abstract}
Work related stress is an increasingly common aspect of modern life and is identified as a considerable risk to employee mental well-being across Europe (Wells, 2011, EASHW, 2014). Numerous psychosocial web based interventions have been developed in response to this. However, targeted psycho-social interventions aimed at enhancing worker resilience and their management of work-related stress are needed if the issue is to be tackled effectively (Wells et al. 2011). This paper documents the development of two digital platform programmes, namely ROSE and DELAROSE, which deliver tailored interventions and act as valuable and accessible supports for workers in the health and social care sectors. The paper also describes the process of transitioning the ROSE into the educational DELAROSE programme in an effort to incentivise workers in these sectors to engage with the programme, and provide them with an accredited academic award from one of three Higher Education Institutions (HEI's) across Europe in recognition of their learning.
\end{abstract}

Keywords: Stress; Health \& Social Care Workers; Online Approaches

\section{Introduction}

The health and social care sector is ranked as one of the most stressful fields in which to work (Wells, 2011). Those working in this area have been found to experience the highest rates of work-related anxiety, stress and depression in any field, as reported by the UK Health and Safety Executive (2014). The highest rates of work-related stress are to be found amongst nurses, workers in caring personal services and welfare and housing associations. In Austria, approximately 65\% of the 350,000 employed in these sectors report at least one work related stress symptom. The European Agency for Safety and Health at Work (2009) found that 30\% of workers in education, health and welfare report feeling stressed at work.

\section{Managing Work Related Stress}

Identifying specific stressor issues and developing mind-body resilience is recognised as important in terms of prevention, self-management and empowerment of employees to work productively until their retirement age (Lazarus and Folkman, 1984; EASHW, 2014). Interventions that build upon and support individuals' resiliency within organisations are increasingly seen as an effective approach to the management and reduction of occupational stress (Ridge et al., 2011).

Interventions can be categorised into person-work interface directed (enhances the congruence between the person and the organisation e.g. role conflict-ambiguity) and organisationally directed so that enhanced employee well-being can be achieved, depending on the design of intervention (Ruotsalainen, 2008). 
MHealth for wellness focuses on providing immediate mobile access to support individuals in the work place for a range of health related problems and provision of health advice (European Commission, 2014). This paper will document the development and provision of a cost- effective and accessible web based programme for health and social care employees to manage their work-related stress - the European Commission funded DELAROSE (Delivering E-Learning Accreditation to Reduce Occupational Stress in Employment) and ROSE (Reducing Occupational Stress in Employment) projects.

\section{European Commission funded projects:}

In 2009, an international consortium, led by Waterford Institute of Technology, Ireland secured funding from the EU to develop an easy access psycho-social online self-help programme for health and social care workers to manage their work-related stress. The project was called Reducing Occupational Stress in Employment (ROSE), and it surveyed health and social care workers across five European countries. As well as measuring their levels of work-related stress, the project also examined what they felt they needed in terms of organisational policies and support in relation to reducing stress at work. Based on this survey work, expertise within the group and a review of the scientific literature, the ROSE online self -help programme was developed.

The ROSE project aimed to provide an online open access and relevant self-help combined person and work-directed stress management programme in order to improve the long-term retention within services of staff in rehabilitation and support services in health and social care.

An important consideration within the ROSE project was financial viability in relation to the 'reach' of the interventions across the sector. Thus ROSE had to be provided to the target group at minimal cost. Having secured EU development and innovation funding to support ROSE, a web based programme platform was used to develop and initially implement an online self-help intervention programme provided freely to the sector, hosted for minimal cost on the project co-ordinator's server.

In addition to this, the team observed that workers across Europe in this sector saw a lack of training and knowledge in managing challenging situations as a source of work stress. They also specified that they would like to receive some formal recognition in relation to their engagement with ROSE.

The team chose to use Moodle, an open source virtual learning environment (VLE) to deliver the ROSE programme. Preliminary feedback from the users indicated that the Moodle format was too plain and not graphical or interactive enough for potential users. This was an important consideration, since the attractiveness of the learning environment encourages engagement with the ROSE programme. Therefore, the ROSE team decided to utilise an 'off the shelf' software package, 'Articulate', to deliver the programme content.

Nimmo (2014) carried out a pilot study to determine the effectiveness of the ROSE program in a small sample of new graduate nurses in a small hospital in upstate New York. The study assessed whether the ROSE programme as an intervention assisted new graduates with their transition into a hospital setting by improving job satisfaction, decreasing stress, and increasing intent to stay. Also, the researcher attempted to determine if the ROSE program is transferrable across cultures, since it was developed in Ireland for a particular population of EU mental-health workers. 
This study highlighted that the ROSE program did indeed improve job satisfaction, decrease stress levels, and improve overall turnover rates in new graduate nurses and highlights that the ROSE program is indeed transferrable across cultures.

\section{Incentivising the use of ROSE through DELAROSE}

One of the findings arising from the ROSE programme was that workers wanted formal recognition of their learning once they had used ROSE. The team felt that extending ROSE into an accredited programme would incentivise workers to engage with the programme and show employers that they had acquired a skills and knowledge base in relation to their professional practice. Employers indicated that they would favour a formal course as a means of meeting health and safety metrics in the work environment.

These findings formed the basis for a further application to the EU for funding to establish ROSE as an accredited and sustainable programme for health and social care workers and to further strengthen programme content. The result became the DELAROSE project, which stands for 'Delivering E Learning Accreditation to Reduce Occupational Stress'. The programme is funded by the Education and Culture Directorate General, Leonardo Da Vinci fund for Lifelong Learning and is due to be completed at the end of October 2015.

Thus DELAROSE provides an opportunity for participants to receive accredited recognition for their learning from a Higher Education Institutions (HEIs). This could contribute to worker mobility while at the same time the programme would remain available for the purpose of personal support. The team have developed a two pronged strategy in this regard. The first is to embrace the concept of the MOOC (Massive Online Open Course) which still allows for free open access. Second is a registration process for those who wish to acquire credits through the ECVET (European Credit system for Vocational Education and Training) and ECTS credit systems.

Using the MOOC concept to deliver DELAROSE offers the opportunity for health and social care workers to fully engage with the programme as a means to manage their personal working environment to be less stressful and then decide post implementation whether they would like to receive credit for their achievements. This is important as there is considerable variability in the sector in terms of educational ability and qualifications and some workers may feel intimidated in having to 'sign up' for the programme from the start (Wells et al., 2011). The MOOC concept offers these workers the chance to use it and thereby build up their confidence towards registering for it. 


\section{DELAROSE Programme Content:}

Person Centred Management of Work-Related Stress:

1) Introduction to Stress

2) Prevention of Stress

3) Learning Ways of Coping with Stress

4) Changing How You Think About Things

5) Giving Feedback
Environment Centred Management of Work-Related Stress:

1) How the Organisation Contributes to Stress

2) Interpersonal Skills

3) Leadership and Motivation

4) Change Management

5) Organisational Policies

The DELAROSE online programme (www.delarose-project.eu) is provided in three languages (English, German and Italian). The content of the programme consists of units of learning divided under two thematic strands; a primary level intervention which consists of work-directed approaches to stress management, and a second strand which is person-directed and which focuses on the management of one's personal stress.

Expected outcomes from DELROSE include the development of a shared understanding of work-related stress in the sector across Europe and the transfer of skills and knowledge between Swiss and EU educational establishments in this field. It will also enhance European vocational education co-operation through utilising European Credit system for Vocational and Education and Training (ECVET) harmonised with the European Credit Transfer System (ECTS). Additionally, it will establish an ECVET and ECTS referenced online multi-lingual programme (English, Italian and German) in work-related stress management that will facilitate the mutual recognition of qualifications between institutions, and provide an access and progression route for workers within the sector.

\section{Conclusion}

Stress and particularly work related stress is now a feature of modern life and the human condition (Wells, 2011). There have been a number of broad based psychosocial web based interventions developed to deal with this and associated mental wellbeing issues, for example

'Hello Brain'; 'MOOD MAP'; 'PRISM' and 'Oiva'. However, sectoral targeted psycho- social interventions to assist workers with work related stress prevention and management are needed if the issue is to be effectively dealt with since particular working environments can create specific stressors not common in other sectors (Wells et al. 2011). Provision of a cost effective occupational health infrastructure that is readily accessible also provides small scale employers with a facility for the establishment of a resilience building work culture. Digital platforms delivering targeted simple programmes, such as ROSE and DELAROSE, can provide a valuable 
and accessible support for worker and employer that enhances the capability of both to manage work related stress.

\section{References}

European Agency for Safety and Health at Work, (2009). European Risk Observatory ReportOSH In figures: Stress At Work - Facts And figures, Luxembourg.

European Agency for Safety and Health at Work (2014). Priorities for Occupational Safety and Health Research 2013-2020 Brussels EASHW.

European Commission (2011). Commission Staff Working Paper Report on the implementation of the European social partners' Framework Agreement on Work-related Stress Brussels EC.

Lazarus, R.S. \& Folkman, S. (1984). Stress, Appraisal and Coping New York Springer.

Nimmo W. (2014). Stress among new graduate nurses. Is the Rose Program a successful intervention in a hospital setting? Unpublished $\mathrm{PhD}$, John Fischer College, Rochester, NY.

Ridge, M., Wells, J.S., Denny, M., Cunningham, J. \& Chalder, T. (2011). Developing a web based stress management intervention for occupational support workers Journal ofMental Health 20(22)185-197.

Ruotsalainen, J., Serra, C, Marine, A. \& Verbeek, J. (2008). Systematic review of interventions for reducing occupational stress in health care workers. Scandinavian Journal of Work, Environment and Health, 34(3)169-178.

Wells, J.S. (2011). Guest Editorial: The Impact of Stress Amongst Health Professionals Journal of Mental Health 20(22)111-114.

Wells, J.S.; Denny, M. \& Cunningham, J. (2011). Work related stress and European policy A comparative exploration of contextual stressors in the rehabilitation sector in five European countries Journal of Mental Health 20:22:165-173. 